\title{
Radioprotection d'un grand chantier de génie civil dans la zone d'exclusion de Tchernobyl
}

\author{
J.P. CARADEC ${ }^{1}$
}

(Manuscrit reçu le 28 février 2005, accepté le 23 août 2005)

RÉSUMÉ Depuis le tragique accident du réacteur $n^{\circ} 4$, les unités 1,2 et 3 de la centrale de Tchernobyl (ChNPP) ont été successivement arrêtées. Or, depuis l'indépendance de l'Ukraine en 1991, la Russie n'assure plus le traitement du combustible irradié ukrainien. L'Ukraine, avec l'aide de la Communauté internationale, a donc envisagé une solution d'attente. En juillet 1999, à l'issue d'un appel d'offres international, le ministère ukrainien, EnergoAtom, retient un consortium de trois entreprises francaises, Framatome, Vinci Construction et Bouygues Travaux Publics, est retenu pour la construction d'un centre de stockage intermédiaire de longue durée du pour la construction d'un centre de stockage intermédiaire de longue durée du
combustible usé des unités 1,2 et 3 , l'« Intérim Storage Facility-2» (ISF-2). La construction démarre en mars 2000. La spécificité de ce chantier de génie civil est son déroulement dans la zone d'exclusion, au voisinage immédiat de la centrale accidentée. Les exigences de sécurité radiologique liées à l'implantation d'un tel chantier dans un environnement contaminé font l'objet de la présente synthèse. Après une description succincte du projet, de son environnement et des dispositions de sécurité radiologique, l'article présente les particularités du chantier. Le contexte local de culture de sûreté, l'article présente les particularités du chantier. Le contexte local de culture de surreté, malentendus entre les acteurs occidentaux et ukrainiens et créer quelques difficultés. Afin de développer une prise de conscience du risque radiologique, l'ensemble du personnel expatrié et ukrainien a reçu une formation pendant toute la durée du chantier. Grâce aux importants moyens engagés et aux actions de prévention, de formation et de contrôle, le chantier s'est déroulé sans problème majeur. L'expérience ainsi acquise peut indubitablement apporter un enseignement profitable pour des ainsi acquise peut in
situations semblables.

ABSTRACT Radioprotection on a large construction site located in the Chernobyl exclusion zone. Since the tragic accident of the reactor $n^{\circ} 4$, units 1,2 and 3 of the Chernobyl Nuclear Power Plant (ChNPP) have been successively stopped. However, since the independence of the Ukraine in 1991, Russia becomes no longer responsible for reprocessing Ukrainian spent fuel. With the support of the International Community, Ukraine agreed to an interim storage solution. In July 1999, following an international invitation to tender, Ukrainian Ministry, EnergoAtom, chooses a consortium of three French companies, Framatome, Vinci Construction and Bouygues Travaux Public, to build an storage facility. Construction of the "Interim Storage Facility-2" (ISF-2) for long term storage of the spent fuel from Chernobyl Units 1, 2 and 3, starts in March 2000. The specific feature of this civil engineering project is its location in the vicinity of the power plant, within the "exclusion zone". The setting up of such a major construction site in a contaminated environment requires a particular attention in radiological safety field. After a brief description of the project, of its environment and radiological safety provisions, this report details the safety measures put in place and the peculiarities of the site. Different safety cultures and occasional misunderstandings between Ukrainian and Western

\footnotetext{
Vinci Construction Grands Projets, 5 cours Ferdinand de Lesseps, 92851 Rueil Malmaison, France.
} 


\begin{abstract}
staff created additional difficulties. In the radioprotection field, the French companies committed significant means and undertook prevention training activities in order to develop awareness of radiological risks by both expatriate and Ukrainian personnel. The experience gained on this site may be described as beneficial in the sense that it teaches lessons for use on future sites in similar conditions.
\end{abstract}

Keywords: Chernobyl / radioprotection / storage / ISF-2 / spent fuel

\title{
1. Le projet
}

Depuis l'indépendance de l'Ukraine en 1991, la Russie n'assure plus ni le retraitement du combustible usé des centrales électronucléaires ukrainiennes, ni le rapatriement du combustible des tranches RBMK (Reactor Bolchoï Mochnosti Kanalynyl). Celui-ci est donc entreposé sur le site des centrales, soit en piscine, soit dans le cœur même des tranches définitivement arrêtées. C'est le cas pour la dernière charge de combustible des tranches 1, 2 et 3 de la centrale de Tchernobyl. Celle-ci, a vu ses tranches arrêtées respectivement en 1991 pour la nº 2, en 1996 pour la $\mathrm{n}^{\circ}$ 1, et en 2000 pour la tranche 3, conformément aux engagements internationaux. Depuis le 15 décembre 2000 la centrale de Tchernobyl ne produit plus d'électricité.

Avec l'aide financière de la Communauté internationale, le gouvernement ukrainien lance, en 1998, un appel d'offres international pour la conception et la construction d'un centre d'entreposage hors sol, sur le site même de la centrale et destiné au combustible usé des trois premières tranches.

En juillet 1999, le ministère ukrainien de l'énergie atomique, « EnergoAtom », retient le projet présenté par le consortium formé de trois entreprises françaises, Framatome (pilote), Vinci Construction (pilote du génie civil) et Bouygues Travaux Publics, ces deux dernières entreprises se groupant ici en « Civil Work Joint Venture » (CWJV).

Financé pour un montant de l'ordre de $80 \mathrm{M} €$, dont environ 35 pour le génie civil, dans le cadre du Nuclear Safety Account, (Union Européenne, USA, Canada, Japon), administré par la Banque européenne pour la reconstruction et le développement (BERD), le projet retenu repose sur le concept technologique NUHOMS (Nuclear Horizontal Monitoring System), d'origine américaine et dont Framatome possède la licence. Cette technologie est alors adaptée aux caractéristiques géométriques du combustible RBMK de la centrale de Tchernobyl.

Le projet «Interim Storage Facility 2 » (ISF-2) se compose de deux ensembles (Fig. 1). 


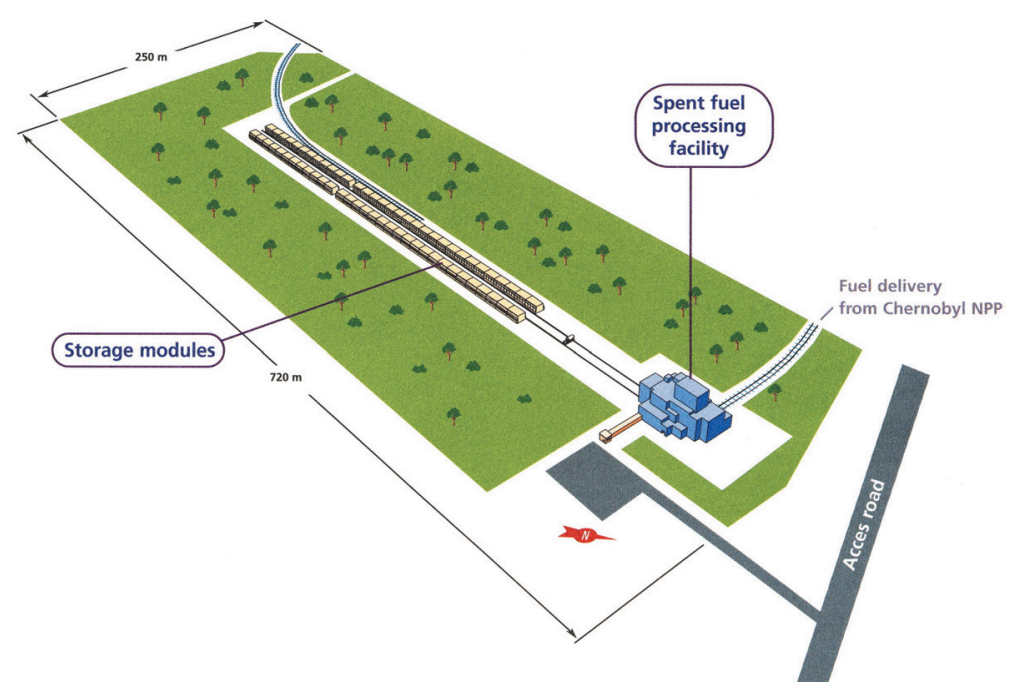

Figure 1 - Projet proposé par le consortium.

Sketch of the project proposed by the consortium.

- Une usine de tri, découpage et conditionnement des assemblages de combustibles dénommée «Spent Fuel Processing Facility» (SFPF). Ces assemblages, d'une longueur de l'ordre de 10 mètres comprennent deux éléments combustibles de 4 mètres environ chacun, auxquels s'ajoute un élément de manutention. Ces éléments sont séparés, par découpage, puis conditionnés sous gaz inerte dans des cartouches étanches, elles-mêmes conditionnées sous gaz dans de grands conteneurs étanches, les « canisters».

- Une structure d'entreposage hors sol est constituée de deux rangées de 29 modules de béton de 4 alvéoles chacun, soit 232 alvéoles destinées à un stockage d'une durée de 100 ans, à raison d'un «canister » par alvéole. Le refroidissement interne de ces dernières est assuré par une convection naturelle au travers de chicanes. Le transfert des « canisters » et leur chargement dans les alvéoles s'effectuent à l'aide d'une machine de chargement-déchargement se déplaçant sur une voie de roulement séparant les deux rangées de modules. Ce sont 3000 tonnes de combustible nucléaire usé et 3000 barres de contrôle qui doivent être stockés dans cet ouvrage et en soulignent l'importance.

Le consortium dispose de 222 semaines pour concevoir, réaliser et mettre en service, sans contrainte radiologique, ce centre de stockage. Les travaux, autorisés 


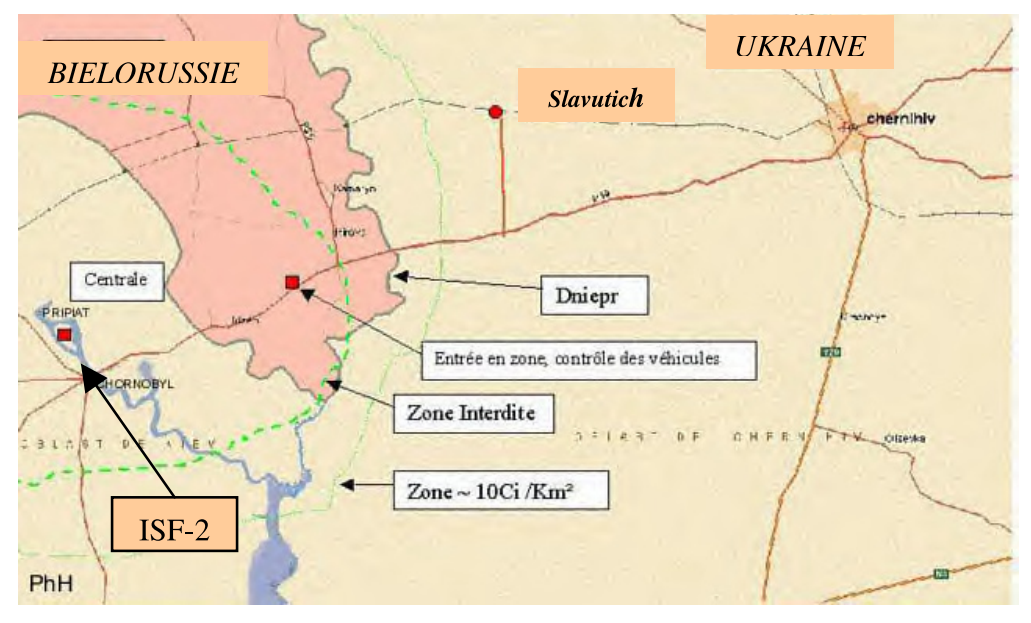

Figure 2 - Situation de la centrale de Tchernobyl et du chantier ISF-2.

Layout of the Chernobyl Nuclear Power Plant and of the ISF-2 site.

par EnergoAtom (National Nuclear Power Utility) débutent en mars 2000 avec pour maître d'ouvrage la centrale de Tchernobyl (Chernobyl Nuclear Power Plant - ChNPP). La maîtrise d'œuvre est assurée par une direction de projet (Project Management Unit - PMU, associant Westinghouse et Chernobyl Nuclear Power Plant).

\section{Le site et son environnement}

Le site retenu pour l'édification de l'ISF-2 se situe au cœur de la « zone d'exclusion », à $2500 \mathrm{~m} \mathrm{du}$ « sarcophage » qui abrite les ruines du réacteur $\mathrm{n}^{\circ} 4$. Entouré de régions boisées et contaminées, il couvre une aire d'environ 17 hectares (Fig. 1).

Cette zone d'exclusion, définie par un cercle de $30 \mathrm{~km}$ de rayon autour de la Centrale, a subi une retombée radioactive hétérogène d'un niveau élevé, estimée de $1,8 \times 10^{13}$ à $3,7 \times 10^{13} \mathrm{~Bq} \mathrm{~km}^{-2}$ pour le ${ }^{137} \mathrm{Cs}$, valeurs de 1986 (MUSU, 1996a). La durée et les conditions de séjour y sont définies par l'administration de la zone d'exclusion (voir Sect. 4) (DRAOTT, 1999 ; Neretine, 2002). La ville voisine, Tchernobyl, également située dans la zone d'exclusion, héberge la plupart des travailleurs locaux. Le personnel expatrié et une partie des travailleurs ukrainiens résident à Slavutich, ville nouvelle, à $72 \mathrm{~km}$ au nord-est du chantier, reliée à la centrale par une route et une voie ferrée qui traverse une partie du territoire Biélorusse (Fig. 2). 
RADIOPROTECTION D’UN GRAND CHANTIER DE GÉNIE CIVIL

Les études effectuées in situ, lors du choix du site, montrent, généralement, une faible migration verticale de la contamination dans les sols où seuls les 15 à 20 premiers centimètres sont concernés par une contamination de ${ }^{137}$ Cs (MUSU, 1996b). Afin de réduire les risques radiologiques, le maître d'ouvrage a entrepris, à l'automne 1999, des travaux d'assainissement par élimination de la végétation contaminée et décapage sur 0,5 mètre d'une grande partie de l'aire réservée à la construction de ISF-2. Cette couverture végétale et ce sol contaminés sont évacués vers des sites d'enfouissement réservés à cette fin, hors du chantier. Le site ainsi remanié est alors clôturé. En dehors de cette aire et des routes refaites, la situation radiologique reste préoccupante.

\section{La situation radiologique initiale du site}

À la remise du site au consortium, le niveau de contamination $\beta$ de la surface, dans sa totalité, est, contractuellement, réputé inférieur à $1,3 \times 10^{5} \mathrm{~Bq} \mathrm{~m}^{-2}$ selon le maître d'ouvrage. Une reconnaissance radiologique complète est toutefois effectuée par le groupement CWJV dès l'ouverture du chantier, permettant l'établissement d'une cartographie de la contamination de surface et du débit de dose sur le site.

La contamination $\beta$ surfacique résiduelle du sol nu est comprise dans la fourchette $10^{2} \leq \mathrm{A} \leq 5 \times 10^{4} \mathrm{~Bq} \mathrm{~m}^{-2}$ avec un niveau moyen sur toute la surface de $3,7 \times 10^{3} \mathrm{~Bq} \mathrm{~m}^{-2}$ (Fig. 3 et Tab. I). Environ $86 \%$ de la surface du chantier est à un niveau de contamination inférieur à $5 \times 10^{3} \mathrm{~Bq} \mathrm{~m}^{-2}$. En 2001, La composition moyenne de la contamination résiduelle se compose essentiellement des produits de fission dont le ${ }^{137} \mathrm{Cs}$, prépondérant, le ${ }^{90} \mathrm{Sr}$, ainsi que des résidus de matière fissile dont les ${ }^{239} \mathrm{Pu}$ et ${ }^{241} \mathrm{Am}$ (et ${ }^{241} \mathrm{Pu}$ ) (Tab. II).

Par ailleurs, les mesures effectuées sur la végétation renaissante mettent en évidence un transfert de la contamination aux plantes, vraisemblablement par voie radiculaire. Avec une radioactivité de niveau très variable mais essentiellement concentrée dans le système foliaire et les tiges de la partie aérienne, ces plantes deviennent le support principal de la contamination apparente sur le site, avec un risque de dispersion à l'automne. L'élimination périodique de ces plantes est la solution adoptée.

D'autre part, bien que le sol soit décapé de la couche supérieure contaminée, les terrassements inhérents à tout chantier de construction mettent parfois à découvert, dans certaines zones du site, une contamination enfouie et occultée jusqu' alors.

Pendant toute la durée des travaux, le débit d'exposition externe à 1 mètre du sol est mesuré en continu par les capteurs d'une station de surveillance située au 


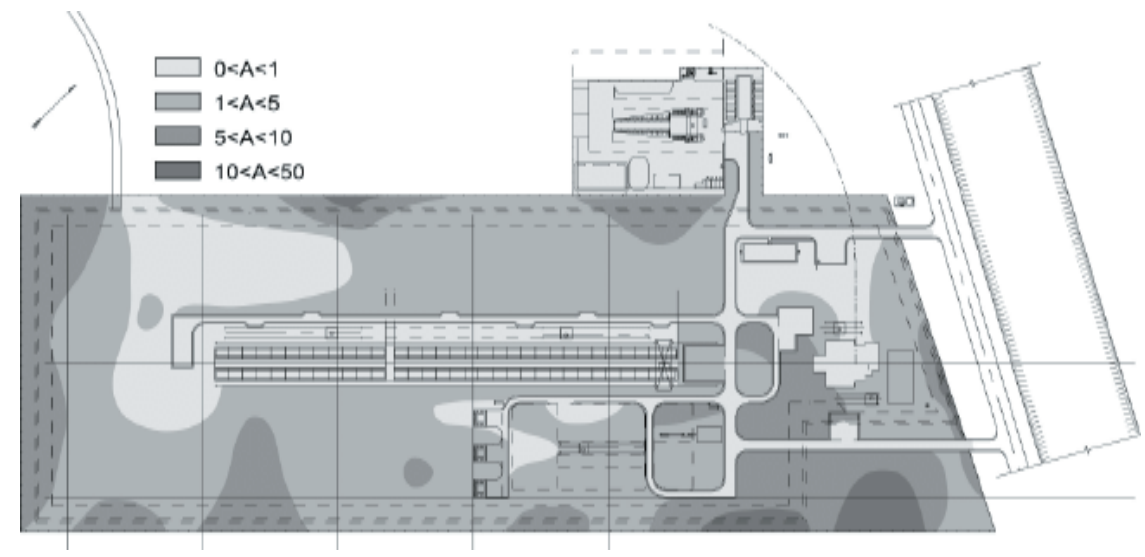

Figure 3 - Contamination $\beta$ surfacique B sur le site ISF-2. Mesures effectuées en juillet 2001. Valeurs en $10^{3} \mathrm{~Bq} \mathrm{~m}^{-2}$.

Surface $\beta$ contamination on ISF-2 site. Measurements carried out in July 2001. Unit: $10^{3} \mathrm{~Bq} \mathrm{~m}^{-2}$.

TABLEAU I

Contamination surfacique sur le site ISF-2. Surface ground contamination on the ISF-2 site.

\begin{tabular}{cc}
\hline Débit d'équivalent de dose en $\mu \mathrm{Sv} \mathrm{h}^{-1}$ & Surface du chantier concernée en $\%$ \\
\hline Irr. $<0,3$ & 58,9 \\
$0,3 \leq$ Irr. $\leq 1$ & 38,8 \\
$1 \leq$ Irr. $\leq 5$ & 2,3 \\
\hline
\end{tabular}

TABLEAU II

Participation relative des différents radionucléides à la composition moyenne de la contamination surfacique dans la zone d'exclusion.

Average composition of the surface ground contamination in the exclusion zone.

\begin{tabular}{cc}
\hline Radionucléide & Teneur \\
\hline${ }^{137} \mathrm{Cs}$ & 63 à $91 \%$ \\
${ }^{134} \mathrm{Cs}$ & 1,4 à $2 \%$ \\
${ }^{90} \mathrm{Sr}$ & 5 à $35 \%$ \\
${ }^{241} \mathrm{Am}$ & $\cong 1 \%$ \\
${ }^{60} \mathrm{Co}$ & $\cong 3 \%$ \\
\hline
\end{tabular}




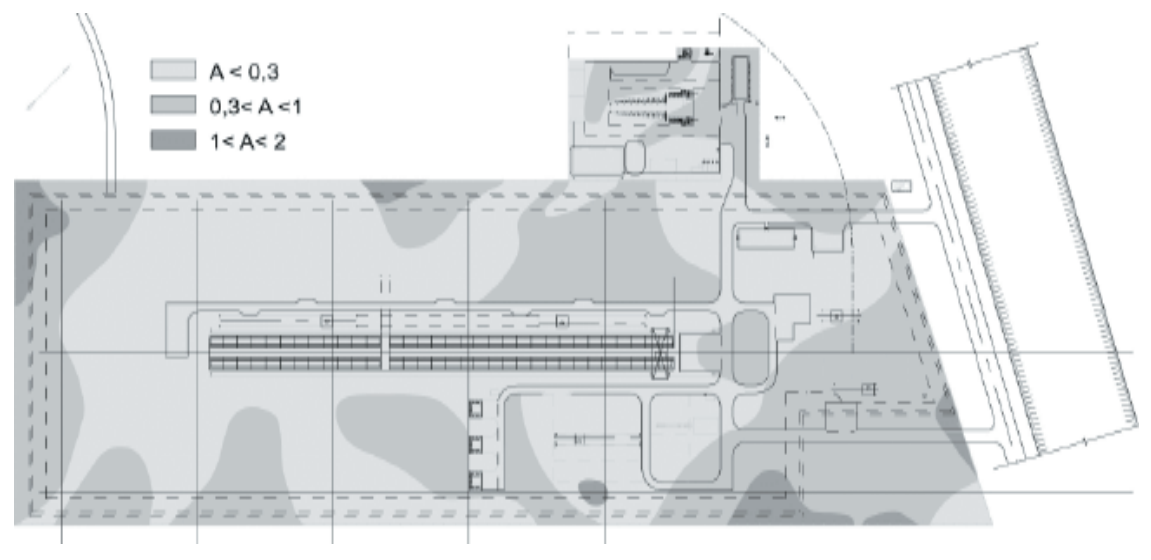

Figure 4 - Débit d'équivalent de dose sur le site ISF-2. Mesures effectuées en juillet 2001. Valeurs en $\mu S v h^{-1} \grave{a} 1 \mathrm{~m}$.

Dose rate at 1 meter above ground on ISF-2 site. Measurements carried out in July 2001. Unit: $\mu \mathrm{Sv} \mathrm{h}^{-1}$ at $1 \mathrm{~m}$.

niveau du bâtiment des bureaux du chantier. Les valeurs observées restent très stables avec un niveau brut moyen de $0,17 \pm 0,03 \mu \mathrm{Sv} \mathrm{h}^{-1}$. Près de $97 \%$ de la zone présente un débit de dose, non corrigé des composantes naturelles, inférieur à $1 \mu \mathrm{Sv} \mathrm{h}^{-1}$ (Fig. 4). La moyenne sur toute la zone est de $0,38 \mu \mathrm{Sv} \mathrm{h}^{-1}$ (Tab. III).

\section{TABLEAU III}

Débit d'équivalent de dose à $1 \mathrm{~m}$ sur le site ISF-2. Dose rate at 1 meter above ground on ISF-2 site.

\begin{tabular}{cc} 
Contamination surfacique $\beta$ en $10^{3} \mathrm{~Bq} \mathrm{~m}^{-2}$ & Surface du chantier concernée en $\%$ \\
\hline Cont. $<1$ & 31,9 \\
$1 \leq$ cont. $\leq 5$ & 53,6 \\
$5 \leq$ cont. $\leq 10$ & 10,9 \\
$10 \leq$ cont. $\leq 50$ & 3,6 \\
\hline
\end{tabular}

La radioactivité artificielle atmosphérique, également mesurée en continu par la station de surveillance du chantier, reste toujours très inférieure aux limites de mesures de l'appareillage de la station $\left(12 \mathrm{~Bq} \mathrm{~m}^{-3}\right.$ en $\beta, 1,2 \mathrm{~Bq} \mathrm{~m}^{-3}$ en $\left.\alpha\right)$. Dans le but de disposer de valeurs interprétables, après décroissance de la radioactivité naturelle, des prélèvements successifs sont regroupés afin d'obtenir des mesures significatives. Celles-ci, fournies par le Laboratoire international de radioécologie de Slavutich, ne dépassent jamais la valeur maximale de $0,01 \mathrm{~Bq} \mathrm{~m}^{-3} \mathrm{de}^{137} \mathrm{Cs}$, 


\section{TABLEAU IV}

Radioactivité artificielle atmosphérique $\beta$ moyenne sur le site ISF-2. Average artificial atmospheric $\beta$ radioactivity on ISF-2 site.

\begin{tabular}{cccc}
\hline Période & Volume $\mathrm{m}^{3}$ & ${ }^{137} \mathrm{Cs} \mathrm{Bq}^{-3}$ & Norme $\beta \mathrm{Bq} \mathrm{m}^{-3}$ \\
\hline juin 2000 & 268 & $6,1 \times 10^{-3}$ & 5 \\
juillet 2000 & 147 & $2,5 \times 10^{-3}$ & $"$ \\
\hline août 2000 & 216 & $10,7 \times 10^{-3}$ & 5 \\
\hline sept. 2000 à nov. 00 & Groupement de prélèvement d'aérosols de la station & $"$ \\
\hline déc. 00 à avril 01 & 14544 & $1,1 \times 10^{-3}$ & $"$ \\
\hline mai 01 à nov. 01 & 21600 & $4,1 \times 10^{-3}$ & $"$ \\
\hline déc. 01 à juil. 02 & 30240 & $2,3 \times 10^{-3}$ & 5 \\
juil. 02 à mars 03 & 31400 & $0,7 \times 10^{-3}$ & $0,2 \times 10^{-3}$ \\
\hline
\end{tabular}

correspondant au niveau observé au tout début du chantier, par grand vent et avec une forte remise en suspension (Tab. IV).

\section{Risques radiologiques sur le site ISF-2}

Même en l'absence de risques radiologiques majeurs, le site ISF-2 est classé zone contrôlée par l'administration ukrainienne car il se situe dans la zone d'exclusion Cette classification implique une évaluation permanente des risques et l'application des règles de radioprotection inhérentes aux travaux en milieu ionisant. À l'issue des investigations et en se référant aux normes ukrainiennes, il est possible de classer les risques radiologiques suivant deux situations :

- une situation que l'on pourrait qualifier de « normale », qui est celle d'un fonctionnement en routine du chantier ;

- une situation exceptionnelle et hypothétique, consécutive à un évènement accidentel extérieur au chantier, nécessitant des dispositions préventives en application du principe de précaution ALARA.

\subsection{Situation de travail dans les conditions «normales»}

Le risque radiologique propre au chantier est essentiellement lié à la nature même du sol, sablonneux et de granulométrie fine. Le vent ou les activités humaines peuvent générer, outre une radio-exposition due à la contamination du sol, une remise en suspension susceptible d'entraîner une radio-exposition supplémentaire externe par contamination cutanée ou interne par inhalation. 
La station de surveillance fournit, en temps réel, la direction et la vitesse du vent ainsi que le niveau de la radioactivité atmosphérique en particules inhalables (avec le ${ }^{137} \mathrm{Cs}$ comme traceur). Ces données, liées à la définition des seuils d'alerte, permettent une organisation rationnelle des procédures de sécurité du chantier.

\subsection{Situations exceptionnelles ou accidentelles}

À ce jour, la possibilité d'un accident à la centrale de Tchernobyl ne peut être écartée, entraînant des conséquences radiologiques graves pour l'environnement. Le site ISF-2 étant placé sous les vents dominants par rapport à la centrale, une procédure d'urgence prend en compte les différentes situations accidentelles envisageables :

- Un effondrement du «sarcophage» qui abrite les ruines de l'unité 4 accidentée et reste toujours considéré comme instable. Différentes études effectuées par plusieurs organismes et instituts (GRS 1995², NIISK $96^{3}, \mathrm{RRC}^{4}$, IREB-ASB ${ }^{5}$...) évaluent la probabilité et les conséquences d'un effondrement de cette structure. Le sarcophage abriterait plus de $50 \mathrm{t}$ de poussières très radioactives (de l'ordre de $2 \times 10^{7} \mathrm{~Bq} \mathrm{~g}^{-1}$ en ${ }^{137} \mathrm{Cs}$ et $4 \times 10^{5} \mathrm{~Bq} \mathrm{~g}^{-1}$ en ${ }^{239+240} \mathrm{Pu}$ dont $75 \%$ de particules inhalables). Un effondrement conduirait, selon les différents scénarios et modèles, à la formation d'un nuage de contaminant avec des conséquences possibles pour le site ISF-2. Ainsi, un rejet de 10 à $100 \mathrm{~kg}$ de ces particules radioactives jusqu'à $100 \mathrm{~m}$ de hauteur entraînerait, avec des vents de 1 à $4 \mathrm{~m} \mathrm{~s}^{-1}$, des doses engagées internes par inhalation, de quelques centièmes au dixième de $\mathrm{Sv}$ au niveau du site ISF-2 (Lavie, 2000 ; IRSN, 2002). Des incertitudes subsistent quant à la valeur de certains paramètres, mais ces évaluations imposent des parades en amont et en aval pour réduire les conséquences radiologiques d'un tel accident.

- Un incendie dans le «sarcophage », accident qui s'est déjà produit, peut également provoquer un accroissement de l'activité atmosphérique par une fuite de particules radioactives pouvant atteindre ISF-2. Un tel incendie d'une durée de 5 heures entraînerait, en effet, une dispersion de plus haute altitude que dans le cas d'un effondrement. D'après les évaluations, une personne exposée sans protection pendant 1 heure à $1 \mathrm{~km}$ sous le vent, recevrait de 10 à $50 \mathrm{mSv}$, selon les conditions météorologiques.

2 GRS : Gesellschaft für Antagen und Reaktorsicherheit - Allemagne.

3 NIISK : Académie ukrainienne des sciences de l'ingénierie (State Research Institute of Building Construction).

4 RRC : Institut Kurtchatov (Radiation/Reactor research Center of Kurtchatov).

5 IREB-ASB : Institut de recherche nucléaires et de l'écologie - Académie des Sciences de Biélorussie. 
- Une excursion critique spontanée dans les ruines de l'unité 4. Les effets directs en resteraient localisés, mais, selon son importance une émission de produits de fission et d'activation volatils ou gazeux, pourrait concerner le site ISF-2.

- Un incendie de la forêt contaminée environnante générerait des fumées et des cendres volatiles susceptibles de survoler et de se déposer, selon les conditions météorologiques, sur le site ISF-2. Elles entraîneraient une contamination générale du site et une augmentation sensible du niveau de la radioactivité atmosphérique.

Tous ces événements accidentels nécessitent, au niveau du chantier ISF-2, une vigilance permanente, associée à des mesures préventives.

Chacun des cas envisagés engendrerait un transfert atmosphérique ponctuel ou continu de contaminant, sous forme d'un nuage radioactif, à faible altitude qui, sur son passage, génèrerait à la fois des effets directs par radio-exposition externe mais aussi une contamination du sol et un risque de radio-exposition interne par inhalation d'aérosols. La coïncidence de pluie au moment du passage d'un tel nuage réduirait les risques de radioexposition interne mais accroîtrait la contamination du sol.

Tous ces scénarios sont susceptibles de concerner le site ISF-2 situé sous les vents dominants de la centrale. Bien que cette dernière dispose d'un système d'alerte, sur le site ISF-2, la vitesse et la direction du vent, fournies par la station de surveillance, sont essentielles car ils déterminent la trajectoire du nuage et l'instant de son éventuelle arrivée au niveau du chantier.

Dans les cas les plus graves, une mise à l'abri immédiate du personnel, sur le chantier, suivie d'une évacuation temporaire ou définitive du site ISF-2 est prévue.

\section{Réglementation et normes applicables sur le site ISF-2}

La réglementation ukrainienne de radioprotection s'appuie sur les valeurs recommandées par la publication 60 de la CIPR (1991) qui ont été transposées dans la législation nationale. Ces valeurs se retrouvent dans le NRBU 97, issu de l'adaptation d'un texte soviétique des années 1986/87 (NRBU, 1997). Bien que dans la région de Tchernobyl ce document reste la référence, les normes applicables sont, en fait, déterminées par l'administration de la zone d'exclusion. Les valeurs sont alors plus restrictives car elle tiennent compte du type et de la nature de la contamination résiduelle in situ et du mode de vie dans une zone où le risque radiologique est considéré comme quasi-permanent (Tab. V). 
RADIOPROTECTION D’UN GRAND CHANTIER DE GÉNIE CIVIL

TABLEAU V

Limites de radio-exposition et de contamination à respecter sur le site ISF-2. Limits of irradiation and contamination to be applied on ISF-2 site.

\begin{tabular}{|c|c|c|}
\hline \multirow{3}{*}{ Limites de radio-exposition } & Exposition externe annuelle sur le chantier ISF-2 & $16 \mathrm{mSv} \mathrm{an}^{-1}$ \\
\hline & Débit de dose effective au poste de travail & $2 \mu \mathrm{Sv} \mathrm{h}^{-1}$ \\
\hline & Débit de dose effective dans les locaux et bureaux & $0,5 \mu \mathrm{Sv} \mathrm{h}^{-1}$ \\
\hline \multirow{2}{*}{$\begin{array}{l}\text { Limites maximales } \\
\text { de contamination atmosphérique }\end{array}$} & Activité Béta & $5,0 \mathrm{~Bq} \mathrm{~m}^{-3}$ \\
\hline & Activité Alpha & $0,0015 \mathrm{~Bq} \mathrm{~m}^{-3}$ \\
\hline \multirow{3}{*}{$\begin{array}{l}\text { Limites maximales } \\
\text { de contamination surfacique }\end{array}$} & Routes et sol & $6,7 \times 10^{4} \mathrm{~Bq} \mathrm{~m}^{-2}$ \\
\hline & Matériel entrant sur le site & $8,4 \times 10^{3} \mathrm{~Bq} \mathrm{~m}^{-2}$ \\
\hline & Locaux et équipement (avant mise en service) & $8,4 \times 10^{3} \mathrm{~Bq} \mathrm{~m}^{-2}$ \\
\hline
\end{tabular}

Tous les travaux effectués dans la zone de Tchernobyl sont soumis à autorisation des instances de la région de Kiev. La zone d'exclusion étant considérée comme "zone contrôlée », le personnel y est classé en « catégorie A » et un document officiel spécifique précise les limites d'irradiation et de contamination pour chaque chantier (Neretine, 2002).

En outre, la zone d'exclusion dispose d'une réglementation du travail particulière, plus restrictive que les dispositions courantes, héritée des années qui ont immédiatement suivi l'accident du réacteur $\mathrm{n}^{\circ} 4$, alors que les conditions radiologiques imposaient des limitations de temps de présence. Ainsi, les horaires sont soit de 36 heures par semaine pour le personnel permanent, soit pour le personnel en alternance, des journées de travail de 10 heures pendant un séjour d'une durée maximale de 15 jours consécutifs suivis de 15 jours de repos hors de la zone d'exclusion (MTU, 1999). Sur le site ISF-2, plus des deux tiers du personnel du chantier appartiennent à cette deuxième catégorie.

Sur le chantier, l'anglais est la langue de travail officiellement retenue tant pour l'oral que pour l'écrit. Afin d'éviter tout malentendu, tous les textes sont cependant traduits en russe. Sur le chantier lui-même, la communication des expatriés avec le personnel local se fait généralement en russe avec l'aide de traducteurs.

\section{Organisation de la radioprotection sur le site}

La radioprotection sur le site incombant contractuellement au groupement CWJV durant les travaux de génie civil, celui-ci met en place un ingénieur expatrié en charge de la radioprotection et de la sécurité du travail et rattaché directement à la direction du chantier. Il est normalement aidé dans cette tache par un ingénieur de sécurité et un agent de radioprotection ukrainiens en postes auprès du sous-traitant 
principal, UkrEnergoBud (UEB). Il a pour tache de s'assurer de la conformité des conditions de travail et de radioprotection avec les réglementations et les normes ukrainiennes comme françaises. Il reste en liaison avec la médecine du travail et les différents organismes ukrainiens et français de radioprotection et de dosimétrie et rédige les différents comptes-rendus. En outre, il met en œuvre et exploite les moyens techniques de contrôle et de mesure fournis par le groupement CWJV, dont la station de surveillance radiologique et les appareils portatifs. Il gère la dosimétrie des travailleurs placés sous la responsabilité directe du groupement CWJV.

La station de mesure et de surveillance assure en continu :

- le contrôle de la radioactivité atmosphérique $\alpha$ et $\beta$ en aérosols inhalables,

- le contrôle de l'irradiation ambiante au niveau de la station,

- la direction et la vitesse du vent.

Le matériel portatif comprend :

- des contaminamètres $\beta \gamma$,

- un appareil de mesure d'irradiation équipé d'une sonde $\gamma$ (cristal d'iodure de sodium - NaI),

- un appareil de prélèvement d'aérosols atmosphériques sur filtre.

Dans le cadre de l'assurance qualité, le responsable s'assure de la vérification et de l'étalonnage systématique de ce matériel auprès de laboratoires extérieurs agréés.

Enfin, à ces taches s'ajoutent l'information préventive et la formation du personnel.

\subsection{Le personnel}

La zone d'exclusion étant considérée comme « zone contrôlée », le personnel y est classé en «catégorie $\mathrm{A}$ ». La vérification de la détention d'un certificat médical attestant de l'aptitude du personnel à cette catégorie incombe au responsable de la radioprotection, aidé dans cette tache par le médecin de l'antenne médicale du site.

L'effectif engagé dans la construction évolue selon les besoins : 350 personnes peuvent être simultanément présentes sur le site qui voit jusqu'à 730 personnes passer dans le mois, compte tenu de l'alternance des équipes en rotation.

Le nombre d'expatriés permanents sur le site est resté, en moyenne, d'une dizaine de personnes, spécialistes ayant tous un rôle d'assistance technique auprès des entreprises locales. En particulier, l'un d'eux a en charge la sécurité du travail et la radioprotection. 
RADIOPROTECTION D’UN GRAND CHANTIER DE GÉNIE CIVIL

Outre le personnel local dépendant directement du groupement de génie civil Vinci - Bouygues (CWJV), la plus grande partie des travailleurs ukrainiens présents sur le chantier dépend d'un sous-traitant principal ukrainien, UkrEnergoBud (UEB), qui dispose lui-même de plusieurs sous-traitants locaux.

\subsection{Information et formation préventive}

Le groupement CWJV a mis l'accent sur l'information et la préparation préventive du personnel. Avant son départ pour l'Ukraine, chaque expatrié suit en France un stage de radioprotection (PR1), obligatoire, auprès d'un organisme agréé. Puis ce personnel reçoit une information donnée avec un maximum de transparence et comprenant une présentation objective des risques radiologiques sur le chantier ISF-2 avec une description des conditions de travail et de vie ainsi que des moyens de sécurité mis en œuvre. En fin d'exposé, tout ce personnel reçoit une brochure rassemblant toutes ces informations (Lavie, 2000).

Sur le site même, une formation complémentaire concerne la totalité des personnes dépendant directement du groupement CWJV, travailleurs expatriés ou locaux, ouvriers comme personnel d'encadrement. Dès leur arrivée sur le chantier, tous assistent à un exposé décrivant les problèmes de sécurité liés à la situation radiologique du site et les normes applicables. Les recommandations concernant la protection contre l'irradiation et la contamination sont commentées. Chacun reçoit un livret d'accueil écrit dans sa langue maternelle, faisant le point des mesures de sécurité à respecter et des moyens mis en œuvre à cette fin.

Dans ce même cadre, le personnel reçoit une formation aux procédures d'urgence applicables dans le cas, même hypothétique, d'un accident radiologique grave. Les dispositifs de surveillance, d'alarme, la procédure d'évacuation sont décrits et expliqués. Les mesures à prendre sont connues de tous et intégrées au plan «Santé et Sécurité » ${ }^{6}$ du chantier. Toute personne présente sur le site et dépendant du groupement CWJV reçoit et dispose en permanence d'une tenue de protection radiologique individuelle utilisable en cas d'alarme. Un opuscule individualisé, toujours dans la langue maternelle de l'intéressé, est remis à chacun. Nominatif, il contient les informations et définit les consignes personnalisées à suivre en cas d'alarme radiologique. On y trouve précisés le lieu de regroupement et de mise à l'abri, l'équipement, et en cas d'évacuation, le moyen de transport prévu. Ces formations sont suivies de rappels et d'exercices d'évacuation périodiques.

\footnotetext{
6 Plan Santé et Sécurité : document interne obligatoire qui regroupe et décrit, avant toute opération de construction sur le chantier, toutes les procédures et moyens mis en œuvre pour assurer la sécurité et l'hygiène.
} 


\section{Antenne médicale}

Une antenne médicale comprenant les moyens de premiers secours aux blessés est intégrée par le consortium dans les moyens de sécurité du site. Un médecin et un infirmier ukrainiens sont présents 7 jours sur 7 dans cette infirmerie bien équipée en matériel médical d'urgence et médicaments importés d'Europe occidentale. Outre les soins classiques, le but de ce dispositif préventif est d'assurer une intervention immédiate auprès d'éventuels blessés et une stabilisation de leur état avant une évacuation vers un hôpital de la région. À cette fin, une ambulance médicalisée reste toujours disponible sur le site.

\section{Le bilan radiologique}

\subsection{Le site}

À l'échelle de temps de la durée des travaux et compte tenu des périodes des radionucléides encore présents, la situation radiologique sur le chantier est restée stable durant toute cette période. Une mise en perspective des valeurs observées avec les valeurs de référence définies par les normes ukrainiennes permet de relativiser les risques auxquels est exposé le personnel.

Ainsi, au cours du chantier, le débit d'équivalent de dose moyen sur le site reste du niveau de l'irradiation externe initiale mentionné au paragraphe 3 , soit de l'ordre du dixième de la valeur maximale de $2 \mu \mathrm{Sv} \mathrm{h}{ }^{-1}$ autorisée au poste de travail et du tiers du maximum de $0,5 \mu \mathrm{Sv} \mathrm{h}^{-1}$ exigé dans les locaux (Tab. V). En effet, toutes les valeurs mesurées restent d'un niveau moyen de l'ordre de 0,2 \pm $0.1 \mu \mathrm{Sv} \mathrm{h}^{-1}$.

Le niveau de la contamination surfacique au sol demeure inférieur à $5 \times$ $10^{3} \mathrm{~Bq} \mathrm{~m}^{-2}$, soit moins du dixième de la norme ukrainienne de $6,7 \times 10^{4} \mathrm{~Bq} \mathrm{~m}^{-2}$ (Tab. V)

La limite maximale de radioactivité atmosphérique $\beta$ autorisée sur le chantier est de $5 \mathrm{~Bq} \mathrm{~m}^{-3}$. Le niveau maximum mesuré sur le site après décroissance de la radioactivité naturelle est de $10^{-2} \mathrm{~Bq} \mathrm{~m}^{-3}$, soit environ le $1 / 500$ de la norme, avec une moyenne pondérée de $1,5 \times 10^{-3} \mathrm{~Bq} \mathrm{~m}^{-3}$ pendant les 34 mois de la durée du chantier (Tab. IV). 


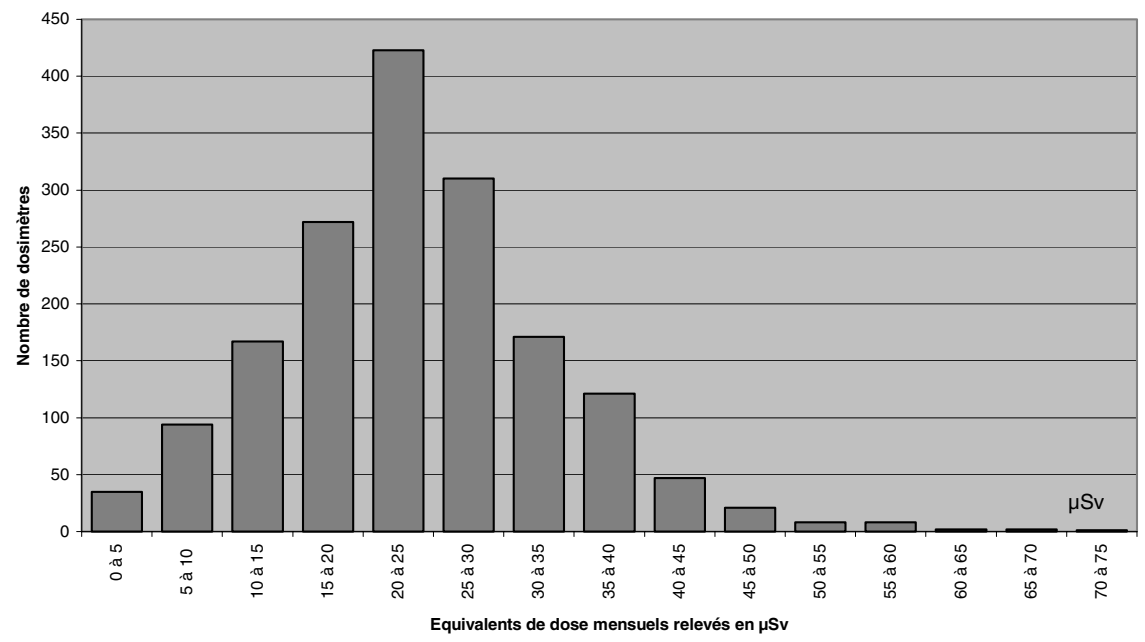

Figure 5 - Distribution Statistique de 1717 équivalents de doses mensuelles mesurés par les dosimètres ukrainiens individuels.

Statistical Distribution of 1717 dose équivalent monthly measures of the personal Ukrainian dosimeters.

\subsection{Le personnel}

\section{Contamination externe}

Quelques cas de contamination de vêtements et d'outillage sont observés lors de travaux de terrassement. Le sable du sol de la zone est souvent le support de cette contamination qui peut atteindre $1,3 \times 10^{4} \mathrm{~Bq} \mathrm{~m}^{-2}$ et n'est généralement pas fixée. Un brossage ou un rinçage à l'eau suffit à l'éliminer de la peau, des vêtements ou du matériel.

\section{Contamination interne}

Les mesures de contamination interne par anthropogammamétrie dont nous avons connaissance, ne montrent pas, chez le personnel expatrié, de valeur supérieure à $2735 \mathrm{~Bq}$ de ${ }^{137} \mathrm{Cs}$, valeur maximale mesurée (Fig. 6) soit le 1/300 de la norme ukrainienne (Tab. VI). Nous n'avons pu disposer des valeurs concernant le personnel ukrainien. 


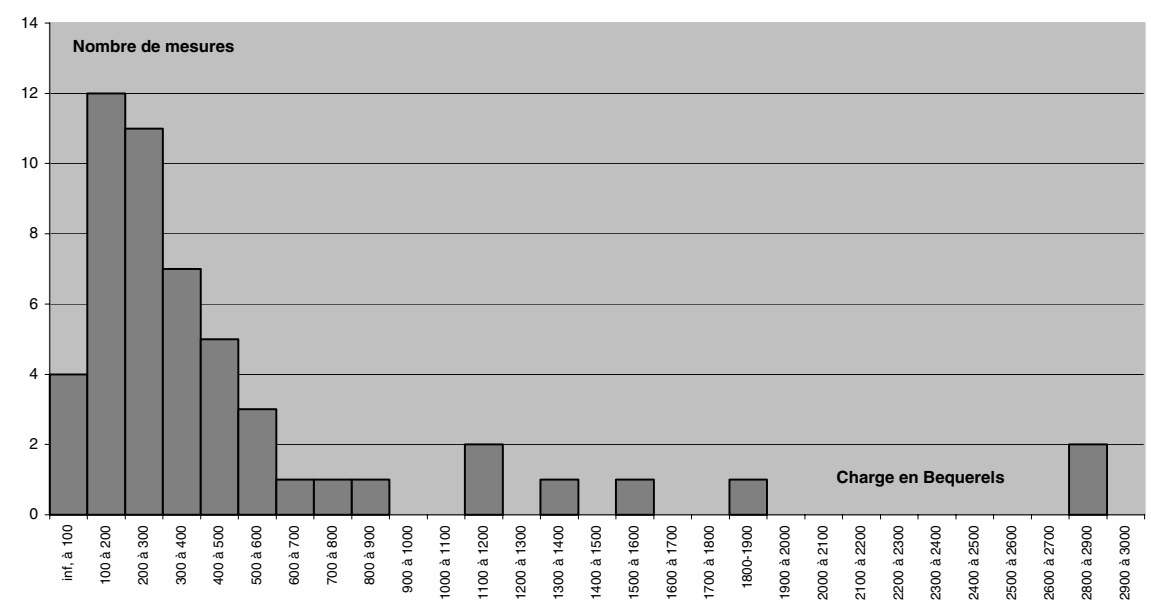

Figure 6 - Distribution Statistique des charges corporelles en ${ }^{137}$ Cs du personnel Vinci-Construction. Statistical distribution of the ${ }^{137}$ Cs body burdens of the Vinci-Construction personnel.

TABLEAU VI

Limites des charges corporelles ukrainiennes et européennes. Ukrainian and European limits of body contents.

\begin{tabular}{ccccccc}
\hline & \multicolumn{2}{c}{ Normes européennes (CIPR 60) } & \multicolumn{3}{c}{ Normes ukrainiennes (NRBU97) } \\
\hline Radionucléide & ${ }^{137} \mathrm{Cs}$ & ${ }^{90} \mathrm{Sr}$ & ${ }^{239} \mathrm{Pu}\left(\mathrm{PuO}_{2}\right)$ & ${ }^{137} \mathrm{Cs}$ & ${ }^{90} \mathrm{Sr}$ & ${ }^{239} \mathrm{Pu}$ \\
Ingestion $(\mathrm{Bq})$ & $4 \times 10^{6}$ & $10^{6}$ & $2 \times 10^{6}$ & $/$ & $/$ & $/$ \\
Inhalation $(\mathrm{Bq})$ & $6 \times 10^{6}$ & $10^{5}$ & $5 \times 10^{2}$ & $10^{5}$ & $3 \times 10^{4}$ & $6 \times 10^{1}$ \\
LDCA $\left(\mathrm{Bq} \mathrm{m}{ }^{-3}\right)$ & $2 \times 10^{3}$ & $6 \times 10^{1}$ & $2 \times 10^{-1}$ & $6 \times 10^{1}$ & $10^{1}$ & $3 \times 10^{-2}$ \\
\hline
\end{tabular}

\section{Irradiation}

Chaque travailleur du site se voit normalement attribuer un dosimètre thermoluminescent individuel fourni par le service de radioprotection de la centrale. Le personnel expatrié porte également son dosimètre national individuel auquel s'ajoute un dosimètre électronique opérationnel. Les dosimètres mensuels individuels, qu'ils soient de type électronique, photographique ou thermoluminescent, d'origine ukrainienne ou française, donnent des résultats moyens très cohérents entre eux. Compte tenu de la diversité des dosimètres individuels, les résultats sont présentés selon leur type et le laboratoire de mesure. 
RADIOPROTECTION D’UN GRAND CHANTIER DE GÉNIE CIVIL

Les valeurs citées ci-dessous sont des valeurs brutes de lecture, ne discriminant pas les composantes des rayonnements subis par les dosimètres, qu'elles soient d'origine naturelle (cosmique, tellurique) ou artificielle.

La dose collective, somme de toutes les doses reçues par l'ensemble des participants pendant une période donnée, ne peut être ici un indicateur du risque radiologique réel car elle ne discrimine pas les doses susceptibles d'un impact sanitaire. La dose moyenne et la dose maximale observées pendant la même période leur sont donc associées.

\section{Dosimétrie opérationnelle ou active (temps réel)}

Les dosimètres opérationnels, de type Dosicard, ont été mis en service au $1^{\mathrm{er}}$ août 2000 et sont uniquement portés par le personnel expatrié présent sur le chantier. Initialisés à chaque arrivée sur le chantier, ils sont lus sur place en fin de journée de travail. Les équivalents de dose mesurés par ces dosimètres sont mémorisés et intégrées quotidiennement par l'appareil lui-même et leur somme en fin de mois fournit la dose mensuelle attribuable au porteur.

Pendant la durée du chantier couvrant la période allant d'août 2000 à mars 2003, l'équivalent de dose totale intégré par l'ensemble du personnel expatrié, mesuré par ce type de dosimètre, est de $11,87 \mathrm{mSv}$ pour 311 valeurs mensuelles et 5920 hommes jours. La moyenne mensuelle est donc de 38,2 $\mu \mathrm{Sv}$ par mois, avec une moyenne par journée de présence sur le site de $2 \pm 1 \mu \mathrm{Sv}$ pour 10 heures de travail. De cette dernière valeur, et en prenant en compte les incertitudes, il est possible de déduire un débit de dose moyen, pour le personnel présent sur le site, de $0,2 \pm 0,1 \mu \mathrm{Sv} \mathrm{h}^{-1}$ et un équivalent de dose de l'ordre de $400 \pm 200 \mu \mathrm{Sv}$ pour 2000 heures de travail.

Un dosimètre témoin du même type, placé au tableau d'accrochage des dosimètres dans le bâtiment des bureaux du site donne, pendant toute la période précitée, un équivalent de dose intégrée de $5 \pm 1 \mu \mathrm{Sv} \mathrm{j}^{-1}$ soit de l'ordre de $0,21 \pm$ $0,04 \mu \mathrm{Sv} \mathrm{h}{ }^{-1}$

La valeur mensuelle maximale observée est de $64 \mu \mathrm{Sv}$, en novembre 2000, pour une personne ayant été présente 25 jours sur le site. Cette valeur maximale correspond à un débit moyen de l'ordre de $0,25 \mu \mathrm{Sv} \mathrm{h}^{-1}$, soit pour 2000 heures de travail dans les mêmes conditions, à un équivalent de dose intégré de $512 \mu \mathrm{Sv}$.

\section{Dosimétrie ukrainienne (lecture différée)}

Cette dosimétrie légale ukrainienne s'effectue, pour le personnel géré par CWJV, à l'aide de dosimètres thermoluminescents de type Harshaw, exploités par le 
service de dosimétrie de la centrale de Tchernobyl. Ces dosimètres sont portés sur le chantier par tous les personnels qui, hors des horaires de travail, les accrochent à un tableau situé dans le bâtiment des bureaux du site. Ces dosimètres ne quittent donc pas la zone d'exclusion, qu'ils soient portés ou non. Les résultats sont fournis par le laboratoire de dosimétrie de la centrale de Tchernobyl avec une incertitude moyenne évaluée à $30 \%$.

Une difficulté dans l'interprétation des résultats de ces dosimètres vient du fait qu'ils restent en permanence dans la zone d'exclusion, sur le chantier ISF-2. Le résultat globalise la dose reçue par le dosimètre durant tout le mois. Pour déterminer l'équivalent de dose individuel attribuable aux personnes durant leur présence sur le site ISF-2, il est nécessaire de pondérer cette valeur par le temps effectif passé par chacune d'elles sur le chantier. Or le problème vient des différents statuts et horaires des personnels, définis par l'administration de la zone d'exclusion et l'employeur. Nous avons retenu ici une présence moyenne de 200 heures par mois, ce qui donne des valeurs par excès, mais allant dans le sens de la sécurité, pour l'essentiel du personnel ukrainien réputé avoir une durée de travail mensuelle effective de 144 heures généralement effectuée en 15 jours.

Les résultats de cette dosimétrie mensuelle pour tout le personnel administré par le CWJV, après les corrections effectuées en conformité avec les hypothèses ci-dessus, quelle que soit son appartenance ou son statut, sont rassemblés sur la figure 5 .

L'équivalent de dose collective brute totale intégrée par les dosimètres gérés par CWJV est de 403,6 mSv, soit $84 \mathrm{mSv}$ par tout le personnel pendant la durée de sa présence effective sur le chantier et pour la période allant de juin 2000 à février 2003. Cette exposition concerne 1717 hommes mois, soit donc une moyenne $0,32 \mu \mathrm{Sv} \mathrm{h}^{-1}$. Cette dernière valeur permet une évaluation de $544 \mu \mathrm{Sv} \mathrm{an}^{-1}$ pour le personnel ukrainien (1700 heures de travail ; NRBU, 1997). La valeur maximale de $710 \mu \mathrm{Sv}$, mesurée en août 2002 correspond, toujours avec les mêmes hypothèses, à un équivalent de dose de l'ordre de $1,7 \mathrm{mSv}^{-1}$.

La figure 5 montre la répartition statistique des 1717 valeurs brutes mesurées. La valeur moyenne est de $235 \mu \mathrm{Sv}$ pour un mois. Avec les mêmes conventions retenues précédemment, nous retrouvons le même équivalent de dose journalier et les expositions annuelles mentionnées précédemment.

\section{Dosimètres nationaux des expatriés (lecture différée)}

Ils sont portés et conservés en permanence par leur titulaire, c'est-à-dire qu'ils ne restent pas sur le chantier ISF-2 en dehors des heures de travail, mais suivent leurs porteurs à leur domicile de Slavutich. Ces dosimètres sont lus par les organismes 
fournisseurs, soit l'Institut protection et de sûreté nucléaire (IPSN), soit l'Office de protection contre les rayonnements ionisants (OPRI). Ces deux organismes, groupés, sont devenus l'Institut de radioprotection et de sûreté nucléaire (IRSN).

Les résultats de cette dosimétrie, gérés par Vinci-Construction, exploitée par l'IPSN et couvrant une période allant de mars 2000 à novembre 2002, ont donné statistiquement, sur plus de 320 mesures, seulement 4 valeurs dépassant le seuil de détection de $200 \mu \mathrm{Sv}$ et ayant atteint le niveau de $250 \mu \mathrm{Sv}$. Chacun de ces cas a trouvé une explication simplement liée à leur durée d'exposition, supérieure à un mois. Il faut noter que toute dose mesurée inférieure à $200 \mu \mathrm{Sv}$ est dite «nulle » par l'IPSN.

En ce qui concerne le personnel géré par l'entreprise Bouygues et porteur de dosimètres OPRI, nous n'avons, à ce jour, pas connaissance des résultats. Il est toutefois raisonnable de penser qu'ils se situent dans la même moyenne que celle du personnel de Vinci-Construction, les conditions de travail étant absolument similaires. Cette hypothèse est d'ailleurs confirmée, comme nous l'avons vu, par les résultats des dosimètres ukrainiens et opérationnels qui restent du même ordre de grandeur pour tout le personnel.

En définitive, quelque soit le type de dosimètre, normalisées à une durée d'un an sur le site afin d'être comparables, les valeurs brutes, composantes naturelles non déduites sont :

dosimétrie opérationnelle (électronique)

$$
1752 \pm 876 \mu \mathrm{Sv} \mathrm{an}^{-1}
$$

dosimétrie ukrainienne (pastilles thermoluminescentes)

$$
2242 \pm 673 \mu \mathrm{Sv} \mathrm{an}^{-1} ;
$$

dosimétrie IRSN (photographique)

$$
\leq 2400 \mu \mathrm{Sv} \mathrm{an}^{-1} \text {. }
$$

À ces niveaux, les valeurs peuvent être considérées comme homogènes et, il est à noter, du même ordre de grandeur que la dose naturelle moyenne annuelle mondiale de $2420 \mu \mathrm{Sv} \mathrm{an}^{-1}$ (cosmique $390 \mu \mathrm{Sv} \mathrm{an}^{-1}$, tellurique $480 \mu \mathrm{Sv} \mathrm{an}^{-1}$, atmosphérique $1260 \mu \mathrm{Sv} \mathrm{an}^{-1}$, ingestion $290 \mu \mathrm{Sv} \mathrm{an}^{-1}$ ) (UNSCEAR, 2000). Compte tenu des incertitudes de mesure sur des niveaux aussi faibles, il devient difficile d'en déduire une évaluation de la composante artificielle, d'autant que nous ne disposons pas d'estimation de la seule composante naturelle dans la zone. Tous ces niveaux restent cohérents avec le niveau général sur le chantier mesuré par la station de surveillance ISF-2, soit $1490 \pm 270 \mu \mathrm{Sv}$ an $^{-1}$. En effet, celle-ci mesure à environ $1,5 \mathrm{~m}$ du sol, sur une dalle de béton, un débit d'équivalent de dose moyen de $0,17 \pm 0,03 \mu \mathrm{Sv} \mathrm{h}^{-1}$, toutes composantes confondues et pendant toute la durée du chantier. Dans la région, les valeurs suivantes sont affichées en permanence $0,20 \mu \mathrm{Sv} \mathrm{h}^{-1}$ au poste de contrôle de la limite de la zone d'exclusion, 
$0,14 \mu \mathrm{Sv} \mathrm{h}^{-1}$ à la frontière biélorusse, $0,16 \mu \mathrm{Sv} \mathrm{h}^{-1}$ à l'hôpital de Slavutich et $0,80 \mu \mathrm{Sv} \mathrm{h}^{-1}$ au bâtiment administratif de la centrale. Si on considère la valeur des $16 \mathrm{mSv}$ an $^{-1}$ préconisée par les normes ukrainiennes (Tab. V), déduite à partir du mélange radioactif de référence $\left({ }^{137} \mathrm{Cs} 81 \%,{ }^{60} \mathrm{Co} 13 \%,{ }^{90} \mathrm{Sr} \mathrm{3,2 \% ,}{ }^{134} \mathrm{Cs} 2,8 \%\right)$, et si l'on compare les niveaux mesurés sur le chantier avec ceux observés dans la nature en général, il est possible d'affirmer que sur ISF-2, la contribution de la radioactivité artificielle à l'équivalent de dose est difficilement discernable et peu significative.

\subsection{Contrôles radiologiques du site, de l'environnement et des matériaux}

Les contrôles systématiques de la nourriture, des logements, des véhicules de transport du personnel et des matériaux de construction, n’ont jamais donné de valeurs supérieures aux normes (Tab. V), voire même significatives. Par contre, il est arrivé que du matériel d'origine indéterminée, dont l'entrée sur le site n'avait pas été signalée par le poste de garde, soit contrôlé contaminé à des niveaux impliquant son élimination immédiate du site. C'est ainsi que des tôles d'acier récupérées dans la zone et présentant une contamination de $2 \times 10^{5} \mathrm{~Bq} \mathrm{~m}^{-2}$ ont du être immédiatement évacuées.

\subsection{Les situations d'urgences}

Les moyens d'alarme mis en place sur le chantier, dans le cadre du plan d'urgence, ont montré leur efficacité lors des exercices périodiques d'évacuation. Le groupement CWJV a la responsabilité de la sécurité de 140 personnes environ qu'il emploie directement et lors de ces exercices généralement bimestriels, chacune d'elles, équipée de sa tenue de protection, applique parfaitement des consignes précises, connues et individualisées. Malgré l'étendue du chantier, l'évacuation du site par ce personnel s'est toujours effectué dans des temps de l'ordre de 10 à 12 minutes suivant le déclenchement de l'alarme.

\section{Les difficultés rencontrées}

\subsection{Le recrutement du personnel}

Du fait de la dramatisation médiatique des conséquences de l'accident de l'unité 4, une première difficulté a été identifiée lors du recrutement du personnel destiné à travailler sur le site. L'appréhension et les craintes du personnel pressenti, expatrié comme local, ont amené le groupement à prendre des mesures de prévention et à relativiser les risques par un effort particulier de formation suivi de rappels périodiques. 


\subsection{Différence de culture de sûreté}

Sur le site, la différence des cultures de sûreté existant entre l'Ukraine postsoviétique et l'Europe de l'Ouest reste très sensible et peut être à l'origine de quelques malentendus et difficultés. Les bouleversements économiques qui ont suivi l'indépendance de l'Ukraine en 1991, après 70 ans de régime soviétique, ont été amplifiés dans la région de Tchernobyl par les conséquences de l'accident. En particulier, nombre d'usines et kolkhozes ont dû fermer, entraînant un chômage endémique, problème jusqu'alors inconnu dans ce pays. Un des effets indirecte de cette déstabilisation est une dégradation et un laxisme dans l'application des règles de sécurité, phénomène reconnu par l'inspection du travail de Kiev, en charge de notre chantier.

Les entreprises sous-traitantes locales ont, au regard de la loi ukrainienne, la responsabilité de la sécurité de leur personnel, mais leur indifférence aux problèmes de sécurité en général et radiologique en particulier a été source de difficultés, comme le montrent les quelques exemples qui suivent.

\subsection{Formation}

Dans les entreprises locales sous-traitantes la formation des travailleurs reste souvent sommaire et se limite généralement, non pas à des explications, mais à l'énumération, sans commentaire, des interdictions et des sanctions entraînant des retenues sur salaire. Tout en étant conscients des limites de notre démarche, nous avons donc tenté de transférer notre culture de la sécurité radiologique et de prodiguer notre rigueur au personnel ukrainien durant plus de deux années, en mettant l'accent sur la prévention et la formation. Si nous trouvons une adhésion et une participation active auprès du personnel ouvrier, ce n'est pas toujours le cas avec l'encadrement local. Celui-ci privilégie souvent la productivité au détriment notamment de la sécurité radiologique. Il banalise le fait nucléaire d'autant qu'il a en partie vécu l'accident du réacteur $\mathrm{n}^{\circ} 4$ et l'époque des fortes doses de radiations et ne se préoccupe plus guère des risques radiologiques actuels qu'il traite souvent avec désinvolture. Il n'apparaît donc pas aussi réceptif aux formations et sa coopération est souvent difficile à obtenir.

D'autre part, l'ingénieur et le technicien de radioprotection ukrainiens présents sur le site chargés de la sécurité classique comme radiologique ne sont restés en fonction que les premiers mois du chantier avant leur licenciement pour raison économique par le sous-traitant principal. La totalité de cette tache reste donc à la seule charge du responsable de sécurité expatrié. 
La partie du plan «Santé et Sécurité » dont la rédaction incombe au soustraitant principal s'est révélée n'être qu'une transcription d'un texte ne concernant pas notre chantier. Malgré des demandes réitérées, aucun autre document n'a été présenté.

\subsection{Dosimétrie individuelle}

Le port d'un dosimètre individuel est obligatoire sur le chantier conformément aux règles applicables dans la zone d'exclusion. Le personnel ukrainien n'en est cependant pas toujours équipé, l'employeur local, par économie, ne le lui fournissant pas systématiquement, ou, au lieu d'être renouvelé chaque mois, le titulaire conserve le même dosimètre durant plusieurs mois consécutifs. On peut aussi noter que beaucoup de travailleurs ne le portent pas, mais cette fois volontairement, car le dosimètre étant susceptible, à leurs yeux, de témoigner d'une dose supérieure au seuil autorisé, ce qui impliquerait leur éviction du site et généralement la perte de leur emploi.

\subsection{Mobilité du personnel}

Les conditions de vie plus que sommaires, le paiement irrégulier et le niveau des rémunérations du personnel des sous-traitants locaux (65 à $80 €$ par mois pour le personnel ouvrier, parfois payés avec 5 mois de retard) induisent peu de motivation mais par contre, une très grande mobilité des ouvriers. Ce phénomène nécessite, toutes les deux semaines, à chaque rotation des équipes, une formation complète des nouveaux arrivants.

\subsection{Organisation médicale locale}

Liée à la baisse d'activité de la centrale, la déliquescence croissante du dispositif d'urgence médicale est constatée lors d'accidents graves sur le chantier ayant entraîné 2 décès. Ces accidents ayant nécessité l'intervention des services de secours de la centrale, l'action de l'organisation médicale ukrainienne en place a été estimée peu fiable et efficace. Après de nombreuses interventions pour tenter d'améliorer le dispositif et afin de réduire les risques en cas d'accident, le consortium n'a pu compter que sur lui-même et a mis en place sur le site une antenne médicale équipée de ses propres moyens d'intervention et d'évacuation puis a recruté médecins et infirmiers.

\subsection{Alcoolisme}

L'alcoolisme, fléau endémique de la région, n'épargne pas le chantier. De nombreux cas d'expulsion de personnel pour alcoolisme ont illustré la réalité du problème qui concerne aussi bien les travailleurs que leur encadrement. 


\subsection{Exercices d'évacuation}

Afin d'organiser des exercices d'évacuation sur le site, un contact a été pris avec les autorités de la centrale qui dispose non seulement des postes de garde mais aussi des moyens de contrôle et de décontamination en sortie de la zone d'exclusion. Le but était de coordonner nos exercices avec les leurs. Il est apparu qu'il y faut au moins 15 minutes suivant un accident avant tout déclenchement d'alarme et qu'en dépit des dispositions prévues, les exercices d'évacuation ne sont jamais effectués.

Aucune des entreprises sous-traitantes locales n'a souhaité participer à nos exercices d'évacuation. D'ailleurs, si chaque personne gérée par le groupement CWJV dispose d'une tenue complète de protection radiologique nominative, le personnel des entreprises locales ne dispose en revanche de rien de tel, malgré nos demandes insistantes et répétées.

\subsection{Utilisation de matériel contaminé}

Toute entrée sur le site de matériel non contrôlé préalablement ou dont l'origine n'est pas parfaitement connue est absolument interdite et cette directive fait partie des consignes données au poste de garde du chantier. Par négligence, cette instruction n'est pas systématiquement appliquée et il faut constater que les entreprises locales ont tendance à compléter leur équipement en puisant sans état d'âme dans le stock des matériels, engins et bungalows trop contaminés pour un usage normal et volontairement abandonnés dans l'environnement de la centrale. Ce genre d'opérations clandestines d'entrée de matériel contaminé sur le chantier s'effectue généralement hors des horaires normaux de travail.

\subsection{Matériel de radioprotection}

Dans le domaine de la sécurité radiologique, le groupement CWJV a importé d'Europe occidentale la quasi-totalité des matériels de surveillance, de contrôle et de protection individuelle, ceux-ci n'étant pas disponibles sur place dans des délais raisonnables, compatibles avec la planification des travaux. De nombreux problèmes ont été rencontrés avec les matériels de la station de surveillance qui se sont révélés inadaptés aux conditions d'utilisation sur le chantier et en particulier aux fortes variations des caractéristiques du courant électrique. Ces ennuis ont exigé de multiples interventions de personnel du constructeur venu de France, mais sans effet durable.

En conclusion, la majorité des difficultés rencontrées dans la mise en œuvre des mesures de sécurité et de protection peut être imputée non seulement au manque de moyens des entreprises locales mais aussi et surtout aux manquements dont font preuve les employeurs locaux envers la réglementation ukrainienne. 


\section{Retour d'expérience}

Les textes définissant les règles de radioprotection dans la zone d'exclusion émanent d'organes nationaux ukrainiens, de la centrale ou de l'administration de la zone. Ils sont précis et témoignent d'une parfaite compétence.

Dès notre arrivée sur le site, des contacts sont pris avec les responsables de la radioprotection de la centrale et du «sarcophage» ainsi qu'avec les différents laboratoires de mesures radiologiques et de dosimétrie de la région. Nos interlocuteurs font toujours preuve d'une grande compétence scientifique et technique, mais déplorent tous leur manque de moyens. Leurs rapports avec les étrangers paraissent parfois ambigus, mêlant souvent, aux premiers contacts, curiosité et méfiance. S'ils sont fiers d'avoir jadis appartenus à un très grand pays, l'Union soviétique, c'est avec une certaine amertume et faute de moyens financiers qu'ils voient des étrangers venir effectuer des travaux qu'ils s'estiment capables de réaliser eux-mêmes. On constate un réel sentiment de frustration, voire d'humiliation. Il est toutefois important et recommandé, afin d'acquérir la confiance et de conserver de parfaites relations avec les organismes de sûreté ukrainiens, de toujours faire référence à la réglementation locale et, dans la mesure du possible, de faire appel aux compétences et aux moyens locaux. Ainsi, le Laboratoire de métrologie de Tchernobyl, agréé par l'État ukrainien, est retenu pour le suivi et l'étalonnage de nos appareils de mesure de radioactivité d'origine française tandis que les différents prélèvements effectués sur le site sont analysés par un laboratoire d'analyse de Slavutich (International Radioecology Laboratory of Slavutich).

Auprès du personnel, les actions préventives sont toujours parfaitement reçues par les expatriés déjà sensibilisés aux risques radiologiques comme aux précautions associées. En revanche, si le personnel ukrainien s'est montré attentif lors des formations, la diversité des niveaux d'instruction allant du primaire au supérieur a rendu parfois la tache délicate. Même si cette formation paraît influencer leur comportement, on observe encore fréquemment un manque de respect de règles de sécurité jugées trop contraignantes, conduisant souvent à un manque de vigilance ou pire, à des imprudences. Les efforts entrepris sur le site pour une prise de conscience des risques et une sensibilisation à la sécurité radiologique, doivent être poursuivis en insistant bien sur les risques encourus et en montrant que la sécurité de chacun repose d'abord sur le respect des recommandations et des règles. Cet effort soutenu doit développer le sens des responsabilités personnelles, inculquer une réelle culture de sûreté radiologique pratique liée aux activités quotidiennes et garantir une radioprotection proportionnée mais efficace. Le maintien et la diffusion de cette culture doivent faire l'objet de rappels par des exposés périodiques. 
RADIOPROTECTION D’UN GRAND CHANTIER DE GÉNIE CIVIL

Bien que chaque employeur soit responsable de la sécurité de son personnel, la majorité des difficultés rencontrées a le plus souvent pour origine la négligence des sous-traitants locaux qui n'accordent qu'un budget minimum à la sécurité radiologique. Une solution peut consister en une prise en charge de la totalité de la sécurité radiologique sur le site par une seule entité indépendante et responsable d'un budget suffisant et unique alloué à ce poste par les toutes les entreprises intervenantes.

Par ailleurs, même si dans la zone d'exclusion les niveaux des salaires locaux sont bonifiés par rapport à ceux pratiqués dans la région, le personnel reste instable. Cette mobilité peut être corrigée par une sécurisation du paiement de salaires plus motivants et une amélioration des conditions de vie du personnel sur le chantier comme en dehors.

La lutte contre l'alcoolisme sur le site trouve en partie sa solution dans l'action menée par l'antenne médicale. Celle-ci se charge des constats d'alcoolémie et effectue chaque jour, à l'ouverture du chantier, le contrôle systématique des personnes dont les postes de travail présentent un risque particulier comme le travail en hauteur et la conduite de véhicules ou d'engins.

Enfin, si nous n'avons aucun problème avec le matériel portatif de radioprotection, d'origine française, le matériel de même provenance équipant la station de surveillance n'a pas la fiabilité attendue car inadapté aux conditions d'un tel chantier. Un cahier de charges beaucoup plus exigeant est nécessaire dans le cas d'une nouvelle utilisation d'une installation de même type.

\section{Conclusion}

Le projet confié au consortium en 1999 prévoit la construction dans un environnement contaminé d'une usine et d'un ensemble de stockage à livrer sans contrainte radiologique. Preuve est donnée de cette faisabilité par le succès de la réalisation de la partie génie civil de l'Interim Storage Facility-2, au cœur de la zone d'exclusion de Tchernobyl, dans de bonnes conditions de sécurité radiologique.

Compte tenu de l'importance du projet, le chantier, l'un des rares effectués par des entreprises étrangères dans la zone d'exclusion, s'est trouvé particulièrement observé par les autorités et les organismes de sûreté ukrainiens. Le groupement CWJV s'est bien évidemment donné les moyens de respecter un niveau de sécurité et de sûreté radiologique susceptible de répondre aux différentes situations à risques envisageables. Après trois années d'activités, les résultats obtenus permettent d'affirmer qu'aucune personne n'a subi, durant sa présence sur le 
chantier ISF-2, une irradiation ou une contamination hors normes. Ce résultat a essentiellement été obtenu par un important effort de prévention et de formation du personnel. La preuve est ainsi apportée de la possibilité de travailler dans de telles conditions, tout en maîtrisant efficacement les risques radiologiques particuliers auxquels les travailleurs peuvent se trouver exposés.

La bonne gestion de la sûreté radiologique ne dépend pas, ici, uniquement de textes réglementaires exemplaires et de la sanction des écarts, mais demeure également dépendante de l'investissement dans les moyens de surveillance et surtout d'une vigilance de tout instant dans une région où la contamination générale de l'environnement reste préoccupante. En effet, si le site ISF-2 peut être considéré comme une enclave sans contraintes radiologiques particulières car sans risque sur le plan sanitaire en situation normale et hors travaux spécifiques, sa localisation au cœur de la zone d'exclusion exige une surveillance permanente du milieu physique et un suivi constant du personnel. Le succès obtenu résulte aussi dans l'effort réalisé pour la formation du personnel par une pédagogie adaptée au contexte local tout en donnant une perception juste et mesurée des risques encourus, entraînant ainsi une implication active du personnel. En dépit des difficultés, nous pouvons estimer que l'objectif d'une bonne maîtrise de la situation radiologique comme d'une gestion efficace de la radioprotection est atteint.

\section{RÉFÉRENCES}

DRAOTT (1999) Direction régionale des activités organisationnelles et techniques de Tchernobyl, Prescriptions sur la sécurité radiologique du personnel dans la zone d'exclusion.

ICRP Publication 60 (1991) 1990 Recommendations of Internationnal Commission on Radiological Protection, Ann. ICRP 21(1-3).

IRSN (2002) Document Institut de radioprotection et de sureté nucléaire, avril 2002, Tchernobyl, 16 ans après.

Lavie J.M. (2000) Vivre à Slavutich et travailler à Tchernobyl, Les risques et leur maîtrise.

MTU (1999) Ministère du travail de l'Ukraine, Code du travail de l'Ukraine. Législation du travail dans la zone d'exclusion, Ordre $n^{\circ}$ 667/3960 du 01.10.1999, Arrêté du cabinet des ministres de l'Ukraine $\mathrm{n}^{\circ} 223$ du 07.02.2000, Arrêté du cabinet des ministres de l'Ukraine $\mathrm{n}^{\circ} 490 \mathrm{du}$ 31.07.2000.

MUSU (1996a) Ministère ukrainien des situations d'urgence, Atlas de la zone d'exclusion de Tchernobyl - Kiev 1996.

MUSU (1996b) Ministère ukrainien des situations d'urgence, Bulletin du Centre d'études radioécologiques de la zone d'exclusion de Tchernobyl, $\mathrm{N}^{\circ} 10$ de 1996, page 21, tableau 11.

Neretine Y.A. (2002) Direction de la Centrale de Tchernobyl, Niveaux de référence de la sécurité radiologique 41П-C du 13-07-02, Définition des normes à l'intérieur de la zone d'exclusion et du chantier ISF-2.

NRBU (1997) Document de l'Etat ukrainien (1997) - Normes de Sécurité radiologiques de l'Ukraine.

UNSCEAR (2000) Rapport du comité scientifique des Nations-Unies pour l'étude des effets des Radiations, Exposures from Natural Radiation Sources, Volume 1, Annexe B, Tableau 31. 